\title{
1 Biological conversion of gaseous alkenes to liquid chemicals
}

3 Shuchi H. Desai ${ }^{1,2}$, Irina Koryakina ${ }^{1,2,3}$, Anna E. Case ${ }^{1}$, Michael D. Toney ${ }^{1, *}$, Shota Atsumi ${ }^{1, *}$

4

$5{ }^{1}$ Department of Chemistry, University of California-Davis, Davis, California, USA.

6

$7 \quad{ }^{2}$ These authors contributed equally to this work.

$8 \quad$ To whom correspondence may be addressed: Email: satsumi@ucdavis.edu and mdtoney@ucdavis.edu

9

$10{ }^{3}$ Present address: Intrexon Corporation, South San Francisco, California, USA.

11

12 Keywords: Biological gas to liquid conversion, Metabolic engineering, Alkenes, Diols 


\section{Abstract}

14 Industrial gas-to-liquid (GTL) technologies are well developed. They generally employ syngas, 15 require complex infrastructure, and need high capital investment to be economically viable.

16 Alternatively, biological conversion has the potential to be more efficient, and easily deployed to 17 remote areas on relatively small scales for the utilization of otherwise stranded resources. The 18 present study demonstrates a novel biological GTL process in which engineered Escherichia coli 19 converts C2-C4 gaseous alkenes into liquid diols. Diols are versatile industrially important 20 chemicals, used routinely as antifreeze agents, polymer precursors amongst many other 21 applications. Heterologous co-expression of a monooxygenase and an epoxide hydrolase in $E$. 22 coli allows whole cell conversion of C2-C4 alkenes for the formation of ethylene glycol, 1,223 propanediol, 1,2-butanediol, and 2,3-butanediol at ambient temperature and pressure in one pot.

24 Increasing intracellular NADH supply via addition of formate and a formate dehydrogenase 25 increases ethylene glycol production titers, resulting in an improved productivity of $9 \mathrm{mg} / \mathrm{L} / \mathrm{h}$ 26 and a final titer of $250 \mathrm{mg} / \mathrm{L}$. This represents a novel biological method for GTL conversion of 27 alkenes to industrially valuable diols. 


\section{Introduction}

Gas-to-liquid (GTL) technologies convert gaseous substrates, typically methane, into liquid

chemicals. Current industrial GTL processes employ catalysts with high heat and pressure (Haynes and Gonzalez, 2014). Heavy capital investment into large, high volume production facilities are required for economic viability, with the corollary requirement that large volumes of gas are constantly available (Haynes and Gonzalez, 2014). Such facilities are full of technical and economic challenges to build and maintain (Fei et al., 2014; Haynes and Gonzalez, 2014). A simpler and potentially cost effective alternative is to use engineered microbes as catalysts for GTL processes (Haynes and Gonzalez, 2014; Hu et al., 2016). A biological GTL system can operate at a smaller scale, at ambient temperatures, and with lower capital costs than conventional GTL systems. This would enable deployment of this technology to remote regions and allow capture of "stranded" gasses, which are an underutilized resource (Fei et al., 2014; Haynes and Gonzalez, 2014). Microorganisms can, in principle, achieve higher conversion efficiency (and substrate selectivity) than conventional GTL because of the enzymatic nature of the conversions (Fei et al., 2014). These factors make a biological GTL system potentially more economically and environmentally attractive than a conventional chemical GTL system (Fei et al., 2014; Haynes and Gonzalez, 2014).

Vicinal diols have broad applications as chemical feedstocks and fuels. They are produced industrially, largely from petroleum through a series of steps including cracking to alkenes, alkene epoxidation, and hydrolysis (Sabra et al., 2016). Ethylene glycol is of particular interest due to its many uses such as polyester fibers, PET (polyethylene terephthalate) plastics, and antifreeze. In 2010, 20 million metric tons of ethylene glycol was produced globally, with an estimated 5-10\% increase in annual consumption (Yue et al., 2012). Industrially, ethylene glycol 
52 production suffers from low specificity $(\sim 80 \%)$ for epoxidation by $\mathrm{O}_{2}$, relatively poor conversion

53 to monoethylene glycol ( 90\%), and high water and energy requirements (Rebsdat and Mayer,

54 2000). This process presents an opportunity for improvement by biological catalysis, since it can

55 potentially overcome all of these shortcomings. Furthermore, biological production can occur at

56 ambient pressure and temperature and without the requirement of harsh chemical catalysts.

57 Larger diols (C3-C4) are also valuable (Burgard et al., 2016; Nakamura and Whited,

58 2003). For example, 1,2-propanediol is used in the food, pharmaceutical, cosmetic, and

59 unsaturated polyester industries (Sullivan, 2000). It is also produced via alkene epoxidation and

60 hydrolysis. Butanediols such as 2,3-butanediol serve as important feedstocks for rubbers,

61 plastics, polymers, pharmaceuticals and insecticides (Gräfje et al., 2000). Ethylene glycol, 1,2-

62 propanediol, 1,3-propanediol, 1,4-butanediol and 2,3-butanediol have been biologically produced

63 from sugars (Burgard et al., 2016; Cam et al., 2015; Chen et al., 2016; Nakamura and Whited,

64 2003; Pereira et al., 2016; Pereira et al., 2015; Sabra et al., 2016).

Some microorganisms naturally metabolize gasses. For example, methanogens generate

66 and methanotrophs catabolize methane, diazotrophs fix nitrogen gas, and photosynthetic

67 organisms fix carbon dioxide (Desai and Atsumi, 2013; Kalyuzhnaya et al., 2015). This study

68 demonstrates that E. coli can be engineered to directly metabolize gaseous C2-C4 alkenes to the

69 corresponding diols, which are stable liquids under ambient conditions.

E. coli was chosen as a biological host because it is genetically tractable with many

71 engineering tools readily available. The alkene metabolizing pathway was constructed with two

72 heterologous enzymes. The first enzyme is a monooxygenase (MO) that converts the alkene to

73 an epoxide, which is subsequently converted to a diol by an epoxide hydrolase (EH) from

74 Agrobacterium radiobacter AD1 (Rink et al., 1997) (Fig. 1A). This combination of enzymes 

87 Organics.

converts gaseous C2-C4 alkenes (ethylene, propylene, 1-butene, cis-2-butene, and trans-2butene) into the corresponding diols (ethylene glycol, 1, 2-propanediol, 1, 2-butanediol, $R, R$-/S,S2,3-butanediol, and meso-2, 3-butanediol).

\section{Materials and Methods}

\subsection{Reagents}

All enzymes were purchased from New England Biolabs. All synthetic oligonucleotides and DNA sequencing services were provided by Euforins. Chemicals for gas chromatography (GC) and High Performance Liquid Chromatography (HPLC) standards were purchased from Sigma Aldrich. Ethylene gas was purchased from Air Gas. Propene, 1-butene, cis and trans 2-butene, ethylene oxide and propylene oxide were purchased from Sigma Aldrich. 1,2-Epoxybutane was purchased from TCI America. cis and trans 2,3-Epoxybutane were purchased from Acros

\subsection{Plasmid construction}

All plasmids and primers are listed in Table S1 and Table S2, respectively. The pAL1219 plasmid was constructed using a $300 \mathrm{bp}$ fragment from pAL1354 and a $1.8 \mathrm{kbp}$ fragment from pSA69 (Atsumi et al., 2008) cut with AatII and AvrII. All other plasmids were constructed using sequence and ligation-independent cloning (SLIC) (Li and Elledge, 2007). The pAL1220

plasmid was constructed using two fragments; the backbone was amplified from pZE12-luc (Lutz and Bujard, 1997) with primers SD11/YT18 and the gene encoding TOM V106A (Canada et al., 2002) was amplified with primers SD177/YT872. The pAL1293 plasmid was constructed using three fragments; the backbone was amplified from pZE12-luc with primers YT40/YT18 and the gene encoding tolulene-4-monoxygenase (T4MO) (Tao et al., 2004) from Pseudomonas 
mendocina KR1 was amplified with primers SD189/SD191 and primers SD187/SD190. The pAL1307 plasmid was constructed similarly to pAL1293 except that the gene encoding T4MO G103S/A107T (Tao et al., 2004) was used. The pAL1354 plasmid was constructed using two fragments amplified from pZE12-luc with primers SD138/SD194 and primers SD12/SD139. The pAL1439 plasmid was constructed using two fragments; the backbone was amplified from pSA69 (Atsumi et al., 2008) with primers YT5/YT6 and the epoxide hydrolase (echA) (Rink et al., 1997) gene, which was synthesized with an optimized codon usage for $E$. coli by Life Technologies, was amplified with primers SD178/SD179. The pAL1434 plasmids were constructed using two fragments; the backbone amplified from pZE12-luc (Lutz and Bujard, 1997) with primers YT40/YT18 and the gene encoding P450 BM3 910A (Meinhold et al., 2005) was amplified with primers SD210/SD211. pAL1456 was constructed using two fragments; backbone amplified from pAL1439 with primers SD199/SD200 and $f d h$ from Candida boidinii (Shen et al., 2011) amplified with primers SD201/SD202. All plasmids are verified by restriction enzyme digest and sequencing.

\section{3. $\mathrm{NAD}^{+} / \mathrm{NADH}$ assay}

The NAD ${ }^{+} / \mathrm{NADH}$ assay was performed by the BioAssay Systems EnzyChrom NAD ${ }^{+} / \mathrm{NADH}$ assay kit (E2ND-100) kit using the manufacturer's protocol.

\subsection{FDH Assay}

Cells $(6.4 \mathrm{~mL})$ were harvested at $4^{\circ} \mathrm{C}, 2,000 \mathrm{~g}$ for $10 \mathrm{~min}$. They were resuspended in $200 \mu \mathrm{L}$ of $10 \mathrm{mM}$ sodium phosphate buffer containing $100 \mathrm{mM}$ beta-mercaptoethanol (BME). Cells were centrifuged once more, resuspended to a final volume of $200 \mu \mathrm{L}$ in the same buffer as above and lysed using a mini bead beater 8 (BioSpec Productis, Inc.) by 4 rounds shaking, 45 seconds each. The crude lysate was centrifuged at $16,000 \mathrm{~g}$ for $30 \mathrm{~min}$ at $4^{\circ} \mathrm{C}$. The cell lysate was mixed with 
$12110 \mathrm{mM}$ sodium phosphate buffer $(\mathrm{pH} 7.5), 1.67 \mathrm{mM} \mathrm{NAD}^{+}, 167 \mathrm{mM}$ formate, and $100 \mathrm{mM}$

122 BME and absorbance was immediately read at $340 \mathrm{~nm}$ to detect NADH formation. NADH was

123 calculated using an extinction coefficient of $6220 \mathrm{M}^{-1} \mathrm{~cm}^{-1}$ (Schute et al., 1976).

\section{$124 \quad 2.5$. Strain preparation}

125 Strains were grown in LB overnight at $37^{\circ} \mathrm{C}$ with the appropriate antibiotics: ampicillin (200 $126 \mu \mathrm{g} / \mathrm{mL})$, kanamycin $(50 \mu \mathrm{g} / \mathrm{mL})$. Production experiments were carried out using a Modified 127 Hutner's Mineral Base (MSB) Medium (Stanier et al., 1966), which consists of the following: 40 $128 \mathrm{mM}$ phosphate buffer, $7.57 \mathrm{mM}\left(\mathrm{NH}_{4}\right)_{2} \mathrm{SO}_{4}, 0.52 \mathrm{M} \mathrm{N}\left(\mathrm{CH}_{2} \mathrm{COOH}\right)_{3}, 1.25 \mathrm{mM} \mathrm{KOH}, 1.16 \mathrm{mM}$

$129 \mathrm{MgSO}_{4}, 0.3 \mathrm{mM} \mathrm{CaCl}{ }_{2} \bullet 2 \mathrm{H}_{2} \mathrm{O}, 0.72 \mu \mathrm{M}\left(\mathrm{NH}_{4}\right)_{6} \mathrm{Mo}_{7} \mathrm{O}_{24} \bullet 4 \mathrm{H}_{2} \mathrm{O}, 4 \mu \mathrm{M}$ EDTA, $23 \mu \mathrm{M}$

$130 \mathrm{FeSO}_{4} \bullet 7 \mathrm{H} 2 \mathrm{O}, 51 \mu \mathrm{M} \mathrm{MnSO}_{4} \bullet \mathrm{H}_{2} \mathrm{O}, 1.25 \mu \mathrm{M} \mathrm{CuSO}_{4} \bullet 5 \mathrm{H}_{2} \mathrm{O}, 0.68 \mu \mathrm{M} \mathrm{Co}\left(\mathrm{NO}_{3}\right)_{2} \bullet 6 \mathrm{H}_{2} \mathrm{O}, 0.23 \mu \mathrm{M}$

$131 \mathrm{Na}_{2} \mathrm{~B}_{4} \mathrm{O}_{7} \bullet 10 \mathrm{H}_{2} \mathrm{O}$. Overnight cultures were inoculated $1 \%$ in MSB medium containing $5 \mathrm{~g} / \mathrm{L}$ yeast

132 extract and $50 \mathrm{~g} / \mathrm{L}$ glucose. Cells were grown at $37^{\circ} \mathrm{C}$, with shaking at $250 \mathrm{RPM}$. Once $\mathrm{OD}_{600}$

133 reached $\sim 0.4$, gene expression was induced with $1 \mathrm{mM}$ isopropyl- $\beta$-D-thio-galactoside (IPTG).

134 At this point, cells were shifted to $30^{\circ} \mathrm{C}$ at $250 \mathrm{RPM}$ for overnight growth. Subsequently, the 135 cells were harvested at 1,500 $\mathrm{g}$ for $10 \mathrm{~min}$ and resuspended in MSB medium without glucose or

136 yeast extract. The samples are centrifuged once more as above and then concentrated 3-fold to

$137 \mathrm{OD}_{600} 6 \pm 1$ in MSB medium.

\subsection{Ethylene oxide and diol production}

139 A $750 \mu \mathrm{L}$ sample was placed into a $3 \mathrm{~mL}$ rubber capped tube (BD vacutainer). Samples were 140 bubbled with ethylene for $5 \mathrm{~s}$ with $1.5 \%$ ethylene (1 psi). For $100 \%$ ethylene, propylene, 1141 butene, cis-2-butene, and trans-2-butene, samples were bubbled for 1 s. Subsequently, samples 142 were incubated at $30^{\circ} \mathrm{C}$ for $24 \mathrm{~h}$ while lying flat on a shaker (350 RPM). Ethylene oxide samples 
143 were spun down at 16,000 $\mathrm{g}$ and the cell supernatant was used for GC analysis. Diol samples

144 were analyzed via HPLC.

145 Epoxide hydrolase assay

146 A $750 \mu \mathrm{L}$ sample was introduced into $3 \mathrm{~mL}$ rubber capped tubes. Epoxide was added to a final

147 concentration of $5 \mathrm{mM}$ and the sample was incubated at $30^{\circ} \mathrm{C}$ for $24 \mathrm{~h}$ in a shaker (350 RPM).

148 After incubation, samples were centrifuged at 16,000 $\mathrm{g}$ and the supernatant was used for GC 149 analysis.

$150 \quad$ 2.7. Ethylene glycol production in the presence of formate

151 Strains were condensed to $1.5 \mathrm{~mL}$ (starting $\mathrm{OD}_{600} \sim 7$ ) from $6.4 \mathrm{~mL}$ of induced cultures and put in

$15210 \mathrm{~mL}$ rubber capped tubes. Initial formate concentration was $5 \mathrm{mM}$, and $1.5 \%$ ethylene was

153 bubbled in at 1 psi for $20 \mathrm{~s}$. The sample was put at $30^{\circ} \mathrm{C}$ in a microplate shaker (350 RPM). After

$15424 \mathrm{~h}, 5 \mathrm{mM}$ formate was added to the samples and ethylene was introduced as described above.

155 Samples were taken at 24 and $48 \mathrm{~h}$, spun down at 16,000 g and cell supernatant was used for

156 HPLC analysis.

\section{2.8. Ethylene glycol production at high cell density}

158 Strains were condensed to $1 \mathrm{~mL}$ (starting $\mathrm{OD}_{600} 57$ for Strain TOF) from $120 \mathrm{~mL}$ of induced

159 cultures and put in a $10 \mathrm{~mL}$ rubber capped tube. Initial formate concentration was $8.3 \mathrm{mM}$, and

$1601.5 \%$ ethylene bubbled in at $1 \mathrm{psi}$ for 20 seconds. The tube was subsequently put at $30^{\circ} \mathrm{C}$ in a

161 microplate shaker (350 RPM). After 6, 12, and $24 \mathrm{~h}, 5 \mathrm{mM}$ formate was added to the samples

162 and ethylene was introduced as described above. Samples $(200 \mu \mathrm{L})$ spun down at 16,000 g and

163 cell supernatant was used for HPLC analysis at 6 and $48 \mathrm{~h}$.

$164 \quad 2.9$. GC analysis 
165 GC-FID was used to quantify oxides. The GC system is a GC-2010 with an AOC-20 S auto 166 sampler and AOC-20i Auto Injector (Shimadzu). The column used was a DB-Wax capillary 167 column (30 m length, 0.32-mm diameter, 0.50- $\mu \mathrm{m}$ film thickness) (Agilent Technologies). The

$168 \mathrm{GC}$ oven temperature was begun at $40^{\circ} \mathrm{C}$, increased temperature at $45^{\circ} \mathrm{C}$ per minute to $225^{\circ} \mathrm{C}$ and 169 held for 3 minutes, and the FID detector was held at $330^{\circ} \mathrm{C}$. The injection volume was $0.5 \mu \mathrm{L}$, 170 injected at a 15:1 split ratio. Helium was used as the carrier gas. 1-Pentanol (100 mg/L) was used 171 as an internal standard.

172 2,3-Butanediol from epoxide feeding was detected using the HP-5 column (30 m length, $1730.25 \mathrm{~mm}$ diameter, $0.25 \mu \mathrm{m}$ film thickness). The GC oven temperature began at $70^{\circ} \mathrm{C}$ and 174 increased to $150^{\circ} \mathrm{C}$ at $40^{\circ} \mathrm{C}$ per minute, and the FID detector was held at $330^{\circ} \mathrm{C}$. Injection volume 175 was $0.5 \mu \mathrm{L}$, injected at a 50:1 split ratio. Helium was used as the carrier gas. 1-Pentanol (100 $176 \mathrm{mg} / \mathrm{L})$ was used as an internal standard.

\section{2.10. HPLC Analysis}

178 To measure formate and diol formation, cell culture supernatant was measured using a 20A high179 performance liquid chromatography (HPLC from Shimadzu) equipped with a differential 180 refractive index detector (RID) 10A and an Aminex fast acid analysis column (Biorad). The 181 mobile phase contained $5 \mathrm{mM} \mathrm{H}_{2} \mathrm{SO}_{4}$, at a flow rate of $0.6 \mathrm{~mL} / \mathrm{min}$ at $60^{\circ} \mathrm{C}$.

\section{Results}

\subsection{Conversion of alkenes to diols}

E. coli does not naturally assimilate gaseous alkenes, therefore heterologous enzymes that convert $\mathrm{C} 2-\mathrm{C} 4$ alkenes to the corresponding oxides were identified. Epoxides are toxic to living

187 organisms and relatively unstable (Ehrenberg and Hussain, 1981). It was therefore expected that 
efficient microbial production of the diols would require rapid in vivo conversion of the nascent epoxide to the diol so that epoxide buildup, and thereby toxicity, does not occur. This can be achieved by coexpression of an EH that rapidly converts epoxides to diols (Fig. 1A).

An EH encoded by echA from A. radiobacter AD1 was identified (Rink et al., 1997). It has been shown to be active in vitro toward a variety of epoxides including ethylene oxide, propylene oxide and 1,2-epoxybutane (Rink et al., 1997). The EH was expressed well in E. coli harboring a synthetic echA gene, which was codon optimized for E. coli. The in vivo activity was tested by feeding $5 \mathrm{mM}$ of each epoxide to $E$. coli expressing echA. The corresponding diols were observed, confirming that EchA is active inside E. coli (Fig. 1B). From $5 \mathrm{mM}$ ethylene oxide $(220 \mathrm{mg} / \mathrm{L}), \sim 4 \mathrm{mM}(184 \mathrm{mg} / \mathrm{L})$ ethylene glycol was produced, while from $5 \mathrm{mM}$ propylene oxide (290 mg/L), $1.3 \mathrm{mM}$ (76 mg/L) of 1,2-propanediol was formed (Fig. 1B). These results are consistent with previous results showing that EchA has better activity on ethylene oxide than propylene oxide in vitro (Rink et al., 1997). The in vitro activity of EchA was reported to be better with 1,2-epoxybutane than ethylene oxide (Rink et al., 1997), but only 0.8 mM 1,2-butanediol (56 mg/L) was formed from $5 \mathrm{mM}$ 1,2-epoxybutane (361 mg/L) (Fig. 1B). Additionally, $0.2 \mathrm{mM}(17 \mathrm{mg} / \mathrm{L})$ and $0.06 \mathrm{mM}(6 \mathrm{mg} / \mathrm{L})$ 2,3-butanediol were produced from 5 mM cis-2,3-epoxybutane and trans-2,3-epoxybutane (361 mg/L), respectively (Fig. 1B).

Decreasing diol conversion with increasing epoxide size may indicate a diffusional barrier to catalysis since in vitro data (Rink et al., 1997) do not show the same trend (Fig. 1B). Regardless, EchA accepts a variety of epoxide substrates and was chosen as the EH for this pathway. Next, enzymes that can epoxidize alkenes were identified (Fig. 2). Some MOs have been reported to perform this reaction, including P450 BM3 mutants (Alcalde et al., 2004; Fasan et al., 2007; Meinhold et al., 2005), tolulene-4-monoxygenase (T4MO) mutants (Tao et al., 2004), and 
211 toluene ortho-monooxygenase (TOM) mutants (Canada et al., 2002; Carlin et al., 2015). Four

212 candidates TOM (V106A) (Canada et al., 2002), T4MO wild type, T4MO (G103S/A107T) (Tao

213 et al., 2004) and P450 BM3 9-10A (Meinhold et al., 2005) were chosen for further

214 characterization based on reported activities and preliminary screening.

215

During the initial screening in rich media (Luria-Bertani Broth and Terrific Broth),

216 inconsistent titers and culture pigmentation were observed within identical replicates. MOs can

217 convert tryptophan in the rich media into indole, which spontaneously generates colored

218 compounds (Canada et al., 2002). Competition between aromatic amino acids and ethylene may

219 cause the inconsistent results. However, strains harboring the MOs were unable to grow in M9

220 minimal medium (Desai et al., 2015). Another minimal medium, modified Hutner's mineral base

221 (MSB) (Stanier et al., 1966), was identified which has a higher iron content than M9 based

222 medium. It was speculated that this medium would be beneficial for MO activity since these

223 enzymes require iron for catalysis. MSB medium enabled strains containing MOs to grow and no

224 culture pigmentation was observed. Thus, MSB was used for further characterization.

225 The promiscuity of the MOs was explored since EH can accept a variety of epoxides in

226 E. coli (Fig. 1B). The epoxide products of the MO reactions with alkenes are unstable, toxic, and

227 volatile which makes their yields difficult to directly quantify. Therefore, the in vivo MO

228 reactions were coupled to $\mathrm{EH}$, yielding the diol, which is stable and easily quantified.

229

Diol formation from $\mathrm{C} 2-\mathrm{C} 4$ alkenes by strains containing both $\mathrm{MO}$ and $\mathrm{EH}$ is presented

230 in Fig. 2. Ethylene, propene, 1-butene, cis-2-butene, and trans-2-butene were tested for diol

231 formation by introducing $100 \%$ of each gas for $1 \mathrm{~s}$ into the headspace of a $3 \mathrm{~mL}$ vacuum tube.

232 These alkenes were chosen because the corresponding diols are important industrial chemicals

233 used in a variety of applications (Sabra et al., 2016). 
When cells were introduced to $100 \%$ ethylene, ethylene glycol was produced by three of 235 the four strains harboring MOs and EH (Fig. 2B). The strain harboring T4MO (Strain T4) 236 produced $30 \mathrm{mg} / \mathrm{L}$ ethylene glycol, while the strains harboring TOM (V106A) (Strain TO) and 237 T4MO (G103S/A107A) (Strain T4m) produced slightly less ethylene glycol (19 and $24 \mathrm{mg} / \mathrm{L})$. 238 Different ethylene gas percentages were screened for their effect on ethylene glycol formation. 239 When $1.5 \%$ ethylene was used, ethylene glycol was produced up to $60 \mathrm{mg} / \mathrm{L}$ (Fig. S1). With 240 propene as a substrate, Strains TO and T4 produced 10 and $11 \mathrm{mg} / \mathrm{L}$ of 1,2-propanediol, 241 respectively. Slightly less 1,2-propanediol (6 mg/L) was observed with Strain T4m (Fig. 2C). 1242 Butene was converted to 1,2-butanediol with yields of $3 \mathrm{mg} / \mathrm{L}, 4 \mathrm{mg} / \mathrm{L}$, and $5 \mathrm{mg} / \mathrm{L}$ by Strains TO, T4, and T4m, respectively (Fig. 2D). cis-2-Butene was converted to $11 \mathrm{mg} / \mathrm{L} R, R$ - and/or $244 S, S-2,3$-butanediol by Strains T4 and T4m. Quantification methods used in this study cannot 245 discriminate $R, R$ - and $S, S$-2,3-butanediol due to the low titer. Very little conversion of cis-2246 butene was observed for Strain TO (Fig. 2E). trans-2-Butene was converted to meso-2,3247 butanediol (Fig. 2F). Strains TO and T4m produced 16 and $13 \mathrm{mg} / \mathrm{L}$ meso-2,3-butanediol, 248 respectively, while Strain T4 produced more (29 mg/L) (Fig. 2F).

The strain harboring P450 BM3 9-10A did not show conversion to any of the diols in this condition. The P450 BM3 9-10A may require more oxygen than other MOs (Meinhold et al., 251 2005). Additionally, P450s generally require the heme precursor $\delta$-aminolevulinic acid 252 (Meinhold et al., 2005) be added to the cell culture for sufficient heme formation and proper 253 enzyme folding. This extra step is not required for the other MOs tested in this study, and may 254 also contribute to the lack of observed activity. Strains harboring the MO genes but not the EH gene were tested for ethylene oxide 256 production from ethylene (Fig. 2G). Strains harboring TOM (V106A), T4MO, and T4MO 
257 (G103S/A107A) produced ethylene oxide (Fig. 2G), confirming ethylene glycol production 258 occurs via ethylene oxide.

\subsection{Effect of NADH Supply on Ethylene Glycol Production}

The production of diols from gaseous alkene in E. coli demonstrated above is promising

262 but the titers are low. From Fig. 1B, it was concluded that EH is unlikely to be the bottleneck

263 since it converts ethylene oxide to ethylene glycol rapidly. Therefore, it was hypothesized that

264 the intracellular NADH supply limits MO turnover and thereby epoxide formation from

265 ethylene. NADH can be regenerated from $\mathrm{NAD}^{+}$if cells are continuously fed rich medium or

266 sugars such as glucose. However, doing so is not beneficial overall since it increases production

267 costs. Instead, an abundant and inexpensive substrate, formate, was chosen as a source of

268 reducing equivalents. Formate can be formed from $\mathrm{CO}_{2}$ via electrolysis ( $\mathrm{Li}$ et al., 2012), and is

269 converted to NADH and $\mathrm{CO}_{2}$ by formate dehydrogenase (FDH) (Schute et al., 1976). The

270 released $\mathrm{CO}_{2}$ can be recycled to formate via electrolysis (Li et al., 2012). Adding an $f d h$ gene to

271 the present strains and feeding formate should increase intracellular NADH and thereby ethylene

272 glycol titers. To test this hypothesis, the $f d h$ gene from Candida boidinii (Shen et al., 2011) was

273 introduced into Strains TO and T4 (Fig. 2) to give Strains TOF and T4F, respectively. FDH

274 activity in Strain TO was confirmed (Fig. 3A).

275

Strains TOF and T4F were used in a $48 \mathrm{~h}$ experiment where ethylene and $5 \mathrm{mM}$ formate

276 were fed at 0 and $24 \mathrm{~h}$ to determine if formate and $f d h$ were beneficial to ethylene glycol

277 production (Fig. 3). In the presence of both $f d h$ and formate, $140 \mathrm{mg} / \mathrm{L}$ ethylene glycol was

278 observed after $48 \mathrm{~h}$ for Strain TOF, while only $40 \mathrm{mg} / \mathrm{L}$ ethylene glycol was produced in Strain

279 TO without formate and $f d h$, corresponding to a 3.5 -fold improvement (Fig. 3). For Strain T4 
280 (without $f d h$ ), the ethylene glycol titer with formate was $53 \mathrm{mg} / \mathrm{L}$ after $48 \mathrm{~h}$, while Strain T4F in 281 the presence of formate gave $105 \mathrm{mg} / \mathrm{L}$, corresponding to a 2-fold improvement (Fig. 3B).

282 However, the ethylene glycol titer of Strain T4F did not change significantly with formate 283 supplementation. The intracellular $\mathrm{NAD}^{+} / \mathrm{NADH}$ ratio was measured to clarify the relationship 284 between intracellular NADH and ethylene glycol productions (Fig. 3C), but no significant trends 285 were observed.

In separate experiments, ethylene and formate were supplied more frequently to the cells.

287 In one experiment, in $70 \mathrm{~mL}$ test tubes, $1.5 \%$ ethylene was bubbled for one hour every $12 \mathrm{~h}$ with $2885 \mathrm{mM}$ formate supplementation, but this did not improve ethylene glycol titers (data not shown).

289 To improve gas exchange, the cells were placed in a petri dish (to increase the gas-liquid 290 interface) inside of a box pressurized with $1.5 \%$ ethylene, but no improvement in ethylene 291 glycol titers was observed (data not shown). Finally, ethylene and $5 \mathrm{mM}$ formate were fed at 6 , 29212 and $24 \mathrm{~h}$ during a $48 \mathrm{~h}$ production period using a $10 \mathrm{~mL}$ glass blood serum vial. After $6 \mathrm{~h}$, 293 Strains TOF produced $80 \mathrm{mg} / \mathrm{L}$ (Fig. 4). Utilizing this method, $250 \mathrm{mg} / \mathrm{L}$ ethylene glycol was 294 achieved after 48 h (Fig. 4), doubling the highest titer obtained from Fig. 3. 295

\section{Discussion}

This study demonstrates a novel, sustainable route for production of industrially

298 important diols. These compounds are routinely utilized as antifreeze agents, solvents, and 299 polymer precursors (Sabra et al., 2016). The active development of microbial diol production 300 (Burgard et al., 2016; Nakamura and Whited, 2003) prognosticates a future with biologically 301 based industrial production of diols. Previously developed biological routes to diols use glucose 302 or glycerol as the feedstock (Burgard et al., 2016; Nakamura and Whited, 2003), while diol 303 production from gaseous alkenes demonstrated herein is an alternative to sugar feedstocks. It is 
also a first step toward biological GTL conversion of underutilized feedstocks such as stranded natural gas which can be converted into ethylene.

The two enzymes chosen here to construct the novel metabolic route proved broadly useful. The EH from A. radiobacter AD1 encoded by echA has activity on a wide range of substrates. It can hydrolyse ethylene oxide, propylene oxide, and 1,2-epoxybutane in E. coli (Fig. 1B). Previously no conversion of cis-2,3-epoxbutane to the diol was observed in vitro (Rink et al., 1997). However, both cis-2,3-epoxybutane and trans-2,3- epoxybutane were successfully converted to 2,3-butanediol in vivo by E. coli expressing echA (Fig. 1B). Strains TO, T4, and T4m, which contain genes for different MOs, showed activity on several alkenes (ethylene, propylene, 1-butene, cis-2-butene, trans-2-butene) as evidenced by the formation of the corresponding diols (Fig. 2). These unprecedented results demonstrate the promiscuity both for the MOs to form epoxides and the $\mathrm{EH}$ to form the respective diols.

Titers for 2,3-butanediol were greater from trans-2-butene than cis-2-butene, even though EH has less activity on the trans than the cis isomer (Fig. 1B and $\mathbf{2 F}$ ). It was hypothesized that the MOs have greater activity with trans-2-butene than with the cis isomer. This would result in higher epoxybutane production in the suspected rate-limiting conversion of alkene to epoxide with the trans compared to the cis isomer, and, therefore, higher overall meso-2,3-butanediol titers.

Several attempts were made to improve the titer for ethylene glycol. A limiting factor was speculated to be low intracellular NADH concentrations with concomitant low MO activity, since the EH efficiently converts ethylene oxide to ethylene glycol (Fig. 1). To increase NADH supply, FDH was introduced into ethylene glycol forming strains and formate was included in the production medium. Strain TOF in the presence of $5 \mathrm{mM}$ formate showed $>50 \%$ improved 
327 ethylene glycol titer compared to Strain TO (no $f d h$ ) (Fig. 3B). Without formate addition to

328 Strain TOF, ethylene glycol titer does not increase after $24 \mathrm{~h}$, suggesting that a supply of

329 reducing equivalents is essential for continuous ethylene glycol production (Fig. 3B). With

330 formate addition to Strain TOF, the ethylene glycol titer increases $\sim 30 \%$ from $24 \mathrm{~h}$ to $48 \mathrm{~h}$. A

331 number of different culture conditions were tested to explore the interaction between substrate

332 and NADH supply. Supplementation with ethylene and formate at $6,12,24$, and 48 h doubled

333 the ethylene glycol titer (Fig. 4). After $6 \mathrm{~h}$ and $72 \mathrm{~h}$, Strain TOF produced $52 \mathrm{mg} / \mathrm{L}$

334 (productivity of $9 \mathrm{mg} / \mathrm{L} / \mathrm{h}$ ) and $250 \mathrm{mg} / \mathrm{L}$ ethylene glycol, respectively (Fig. 4). Thus, a source of

335 reducing equivalents for regenerating $\mathrm{NADH}$ is crucial for continuous ethylene glycol

336 production. This follows from the fact that one equivalent of NADH is consumed in the MO

337 reaction for every equivalent of diol formed.

The diol titers and productivities reported here are low compared to titers achieved with

339 sugars as a substrate (Pereira et al., 2016; Pereira et al., 2015). Nevertheless, the results presented

340 in this study are encouraging when compared to other GTL systems. Methanotrophs, a natural

341 GTL platform, can be engineered to convert methane into liquid chemicals (Haynes and

342 Gonzalez, 2014), yet working with them is challenging because they are difficult to manipulate

343 genetically, grow slowly, and have low yields (Henard et al., 2016). Despite these challenges,

344 one group demonstrated lactate production $(8 \mathrm{mg} / \mathrm{L} / \mathrm{h})$ from a methanotrophic bacterium

345 (Methylomicrobium buryatense) using a bioreactor (Henard et al., 2016). Another group

346 engineered an archaeal methanogen to produce acetate $(590 \mathrm{mg} / \mathrm{L}$ at $5 \mathrm{mg} / \mathrm{L} / \mathrm{h})$ with methane as

347 a sole carbon source (Soo et al., 2016). Photosynthesis is nature's most prevalent gas capturing

348 system, harnessing $\mathrm{CO}_{2}$ to produce biomass. Several groups have metabolically engineered

349 cyanobacteria for chemical production from $\mathrm{CO}_{2}$ (Desai and Atsumi, 2013). For example, with 
Synechococcus elongatus PCC 7942, a productivity of $36 \mathrm{mg}$ sucrose $/ \mathrm{L} / \mathrm{h}$ from $\mathrm{CO}_{2}$ was achieved (Ducat et al., 2012). Additionally, S. elongatus has been engineered to produce 2,3butanediol with a productivity of $5 \mathrm{mg} / \mathrm{L} / \mathrm{h}$ (Oliver et al., 2013).

Converting methane or $\mathrm{CO}_{2}$ into complex chemicals requires carbon-carbon bond formation which is an energy intensive process. By providing $E$. coli with gaseous alkenes, this difficult and energy intensive step is by-passed, allowing facile production of valuable diols (Sabra et al., 2016). The best ethylene glycol productivity reported here is $9 \mathrm{mg} / \mathrm{L} / \mathrm{h}$ (Fig. 4), which surpasses the biological production of diols from cyanobacteria and acetate from methanotrophs.

The pathway presented here is industrially relevant and contributes to the development of industrially viable metabolic routes for conversion of gaseous substrates into valuable liquid chemicals. Since Fisher-Tropsch technologies to convert methane to liquid fuels require complex facilities, with associated high costs, many companies (e.g., LanzaTech, Intrexon, Calysta, etc) are acknowledging the benefits of biological production and investing in its development. Further optimization, particularly of the MO activity toward alkene epoxidation, may develop this new pathway into a viable industrial process.

\section{Acknowledgments}

This work was supported by the REMOTE program of the ARPA-E (Award: DE-AR0000429).

We would like to thank Thomas K. Wood (Pennsylvania State University) for providing TOM and T4MO mutants and Frances H. Arnold (California Institute of Technology) for providing P450 BM3 mutants. We are grateful to Rebecca E. Parales, D. Alex Carlin and Justin B. Siegel for insightful discussions. 


\section{References}

Alcalde, M., et al., 2004. Colorimetric high-throughput assay for alkene epoxidation catalyzed by cytochrome P450 BM-3 variant 139-3. J Biomol Screen. 9, 141-146.

Atsumi, S., et al., 2008. Non-fermentative pathways for synthesis of branched-chain higher alcohols as biofuels. Nature. 451, 86-89.

Burgard, A., et al., 2016. Development of a commercial scale process for production of 1,4butanediol from sugar. Curr Opin Biotechnol. 42, 118-125.

Cam, Y., et al., 2015. Engineering of a Synthetic Metabolic Pathway for the Assimilation of (d)Xylose into Value-Added Chemicals. ACS Synth Biol. doi:10.1021/acssynbio.5b00103.

Canada, K. A., et al., 2002. Directed evolution of toluene ortho-monooxygenase for enhanced 1naphthol synthesis and chlorinated ethene degradation. J Bacteriol. 184, 344-349.

Carlin, D. A., et al., 2015. Biocatalytic conversion of ethylene to ethylene oxide using an engineered toluene monooxygenase. Chem Commun (Camb). 51, 2283-2285.

Chen, Z., et al., 2016. Metabolic engineering of Corynebacterium glutamicum for the de novo production of ethylene glycol from glucose. Metab Eng. 33, 12-18.

Desai, S. H., Atsumi, S., 2013. Photosynthetic approaches to chemical biotechnology. Curr Opin Biotechnol. 24, 1031-1036.

Desai, S. H., et al., 2015. Isobutanol production from cellobionic acid in Escherichia coli. Microb Cell Fact. 14, 52.

Ducat, D. C., et al., 2012. Rerouting carbon flux to enhance photosynthetic productivity. Appl Environ Microbiol. 78, 2660-2668.

Ehrenberg, L., Hussain, S., 1981. Genetic toxicity of some important epoxides. Mut Res. 86, 1113. 
Fasan, R., et al., 2007. Engineered alkane-hydroxylating cytochrome P450(BM3) exhibiting nativelike catalytic properties. Angew Chem Int Ed Engl. 46, 8414-8418.

Fei, Q., et al., 2014. Bioconversion of natural gas to liquid fuel: opportunities and challenges. Biotechnol Adv. 32, 596-614.

Gräfje, H., et al., 2000. Butanediols, Butenediol, and Butynediol. Ullmann's Encyclopedia of Industrial Chemistry. 6, 407-414.

Haynes, C. A., Gonzalez, R., 2014. Rethinking biological activation of methane and conversion to liquid fuels. Nat Chem Biol. 10, 331-339.

Henard, C. A., et al., 2016. Bioconversion of methane to lactate by an obligate methanotrophic bacterium. Sci Rep. 6, 21585.

Hu, P., et al., 2016. Integrated bioprocess for conversion of gaseous substrates to liquids. Proc Natl Acad Sci U S A. 113, 3773-3778.

Kalyuzhnaya, M. G., et al., 2015. Metabolic engineering in methanotrophic bacteria. Metab Eng. $29,142-152$.

Li, H., et al., 2012. Integrated electromicrobial conversion of $\mathrm{CO}_{2}$ to higher alcohols. Science. $335,1596$.

Li, M. Z., Elledge, S. J., 2007. Harnessing homologous recombination in vitro to generate recombinant DNA via SLIC. Nat Methods. 4, 251-256.

Lutz, R., Bujard, H., 1997. Independent and tight regulation of transcriptional units in Escherichia coli via the LacR/O, the TetR/O and AraC/I1-I2 regulatory elements. Nucleic Acids Res. 25, 1203-10.

Meinhold, P., et al., 2005. Direct conversion of ethane to ethanol by engineered cytochrome P450 BM3. Chembiochem. 6, 1765-8. 
419 Nakamura, C. E., Whited, G. M., 2003. Metabolic engineering for the microbial production of

420

421

422

423

424

425

426

427

428

429

430

431

432

433

434

435

436

437

438

439

440

\section{1,3-propanediol. Curr Opin Biotechnol. 14, 454-459.}

Oliver, J. W., et al., 2013. Cyanobacterial conversion of carbon dioxide to 2,3-butanediol. Proc Natl Acad Sci U S A. 110, 1249-1254.

Pereira, B., et al., 2016. Efficient utilization of pentoses for bioproduction of the renewable twocarbon compounds ethylene glycol and glycolate. Metab Eng. 34, 80-87.

Pereira, B., et al., 2015. Engineering a novel biosynthetic pathway in Escherichia coli for production of renewable ethylene glycol. Biotechnol Bioeng. 113, 376-383.

Rebsdat, S., Mayer, D., 2000. Ethylene Glycol. Ullmann's Encyclopedia of Industrial Chemistry. $13,531-544$.

Rink, R., et al., 1997. Primary structure and catalytic mechanism of the epoxide hydrolase from Agrobacterium radiobacter AD1. J Biol Chem. 272, 14650-14657.

Sabra, W., et al., 2016. Microbial cell factories for diol production. Adv Biochem Eng Biotechnol. 155, 165-97.

Schute, H., et al., 1976. Purification and properties of formaldehyde dehydrogenase and formate dehydrogenase from Candida boidinii. Eur J Biochem. 62, 151-160.

Shen, C. R., et al., 2011. Driving forces enable high-titer anaerobic 1-butanol synthesis in Escherichia coli. Appl Environ Microbiol. 77, 2905-2915.

Soo, V. W., et al., 2016. Reversing methanogenesis to capture methane for liquid biofuel precursors. Microb Cell Fact. 15, 11.

Stanier, R. Y., et al., 1966. The aerobic pseudomonads: a taxonomic study. J Gen Microbiol. 43, $159-271$. 
441 Sullivan, C. J., 2000. Propanediols. Ullmann's Encyclopedia of Industrial Chemistry. 30, 233-

$442 \quad 240$.

443 Tao, Y., et al., 2004. Altering toluene 4-monooxygenase by active-site engineering for the

$444 \quad$ synthesis of 3-methoxycatechol, methoxyhydroquinone, and methylhydroquinone. J

$445 \quad$ Bacteriol. 186, 4705-13.

446 Yue, H., et al., 2012. Ethylene glycol: properties, synthesis, and applications. Chem Soc Rev. 41, $447 \quad 4218-44$.

448 


\section{A}

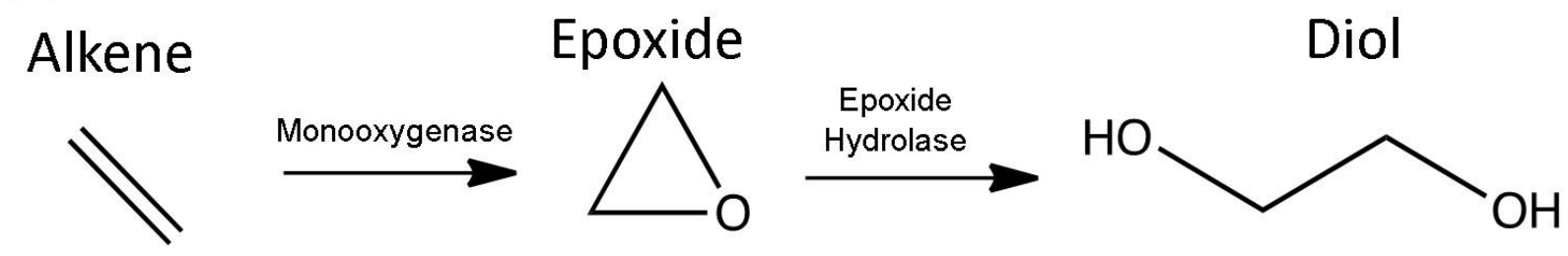

B 6

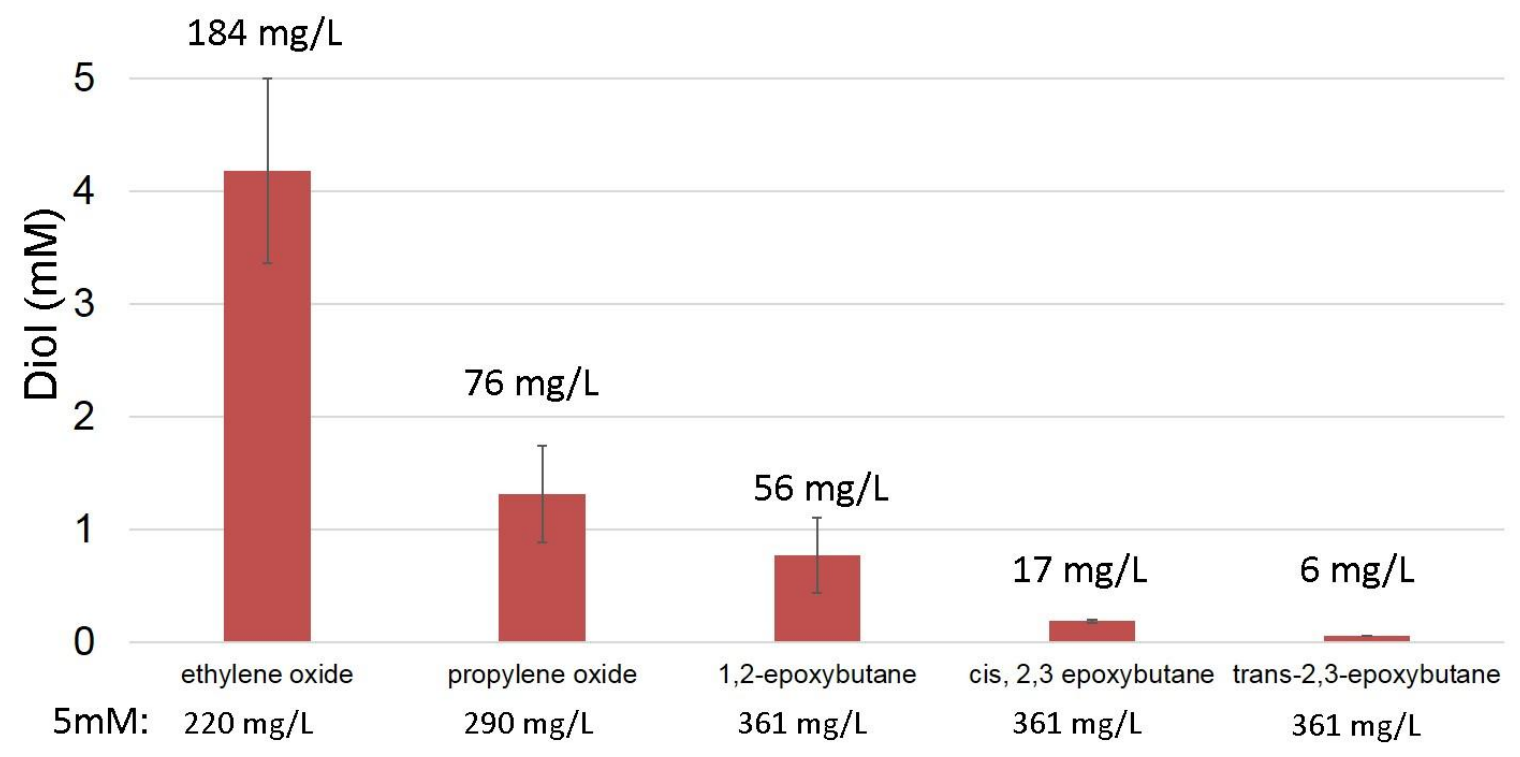

Figure 1. Characterizing enzymes for diol production. A. Reaction scheme for alkene to diol

452 production with ethylene glycol shown as an example B. In vivo conversion of individually fed

453 epoxides to diols. Here, $5 \mathrm{mM}$ of different epoxides were fed to strains expressing the epoxide

454 hydrolase gene. Numbers below epoxides represent mass concentration $(\mathrm{mg} / \mathrm{L})$ for the initial 5

$455 \mathrm{mM}$ solution, while numbers above the bars represent diol formation. In a negative control

456 (empty vector), no detectable peaks were observed. $\mathrm{N}=3$; error bars represent standard

457 deviations. 


\begin{tabular}{|c|l|}
\hline Number & $\begin{array}{l}\text { Monooxygenase } \\
\text { (MO) }\end{array}$ \\
\hline 1 & TOM V106A \\
\hline 2 & T4MO \\
\hline 3 & T4MO G103S/A107T \\
\hline 4 & P450 BM3 910-A \\
\hline
\end{tabular}

B
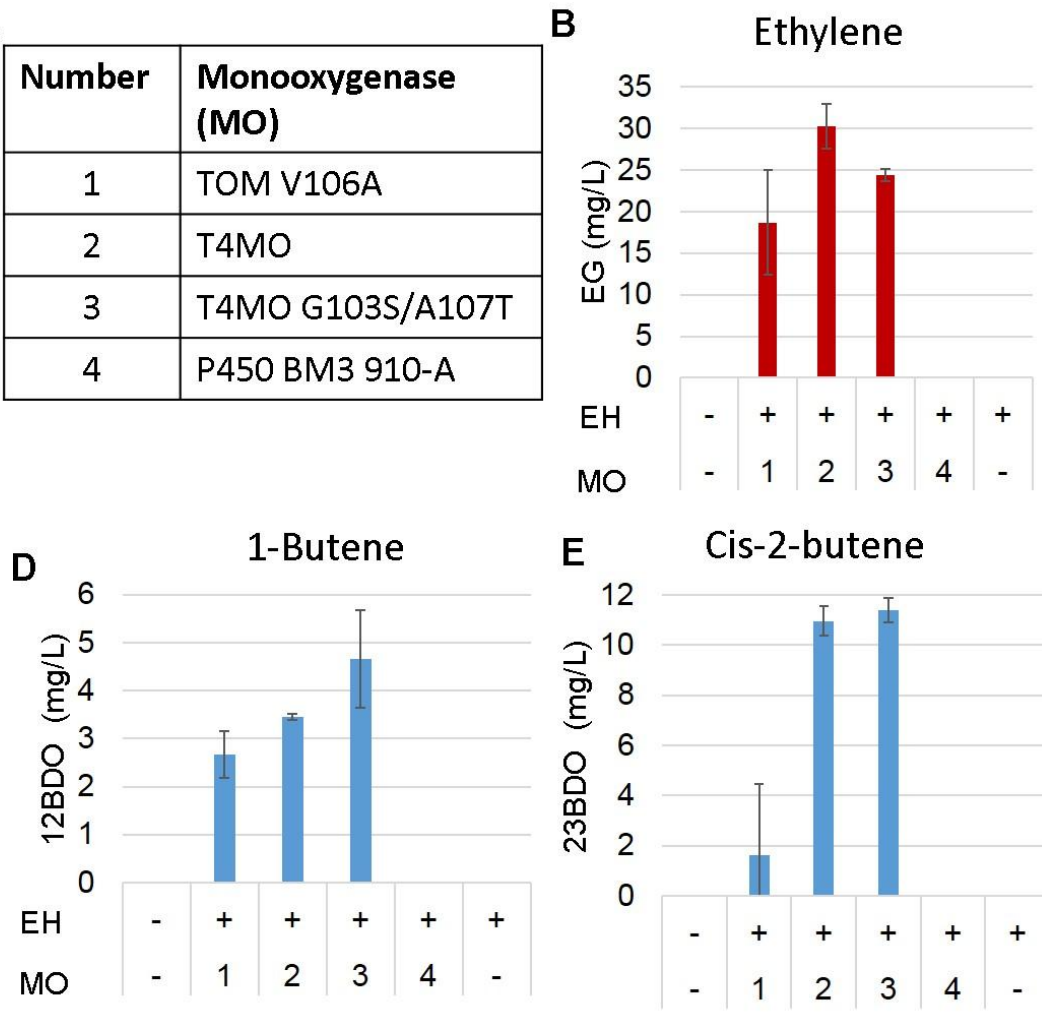

E

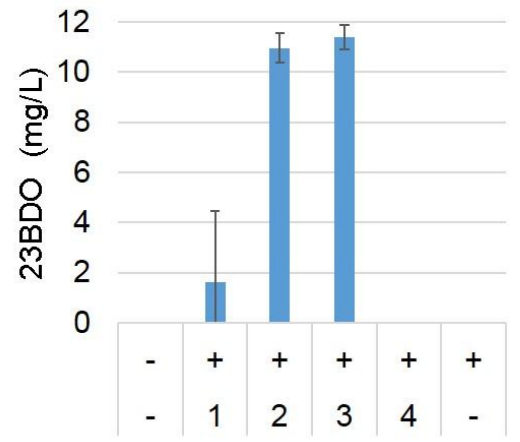

C Propene

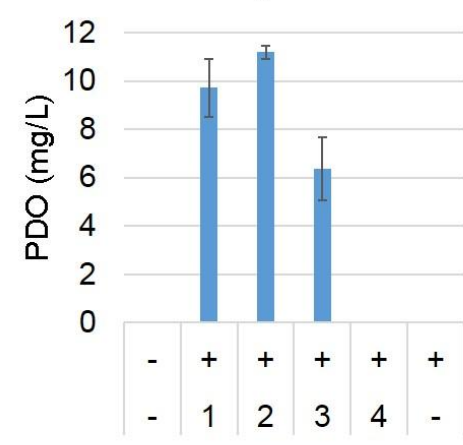

$\mathbf{F}$

Trans-2-butene

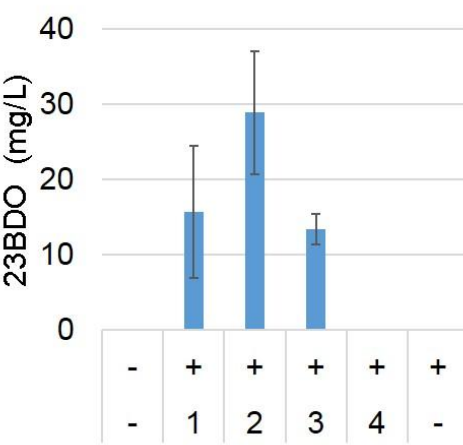

G

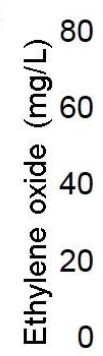

$\mathrm{EH}$

MO

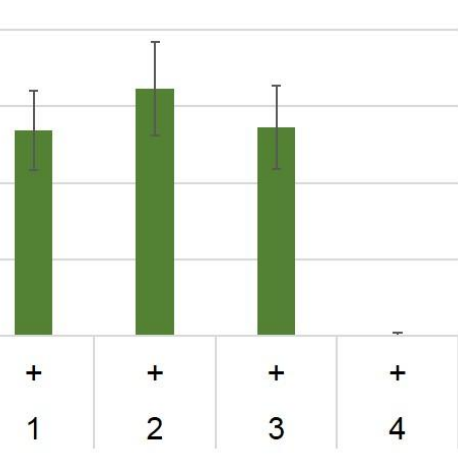

Figure 2. Diol production from different gaseous alkenes. Strains expressing both the monooxygenase (MO) and epoxide hydrolase (EH) genes were bubbled with different gaseous alkenes, and diol production was determined after $24 \mathrm{~h}$. Pure alkene gas was introduced into the headspace for 1 second. A. Monooxygenase numbering. B. Ethylene glycol (EG) production from ethylene C. 1,2-Propanediol production (PDO) from propene. D. 1,2-Butanediol production

467 from ethylene in strains without $\mathrm{EH}$. N=3; error bars represent standard deviations. 


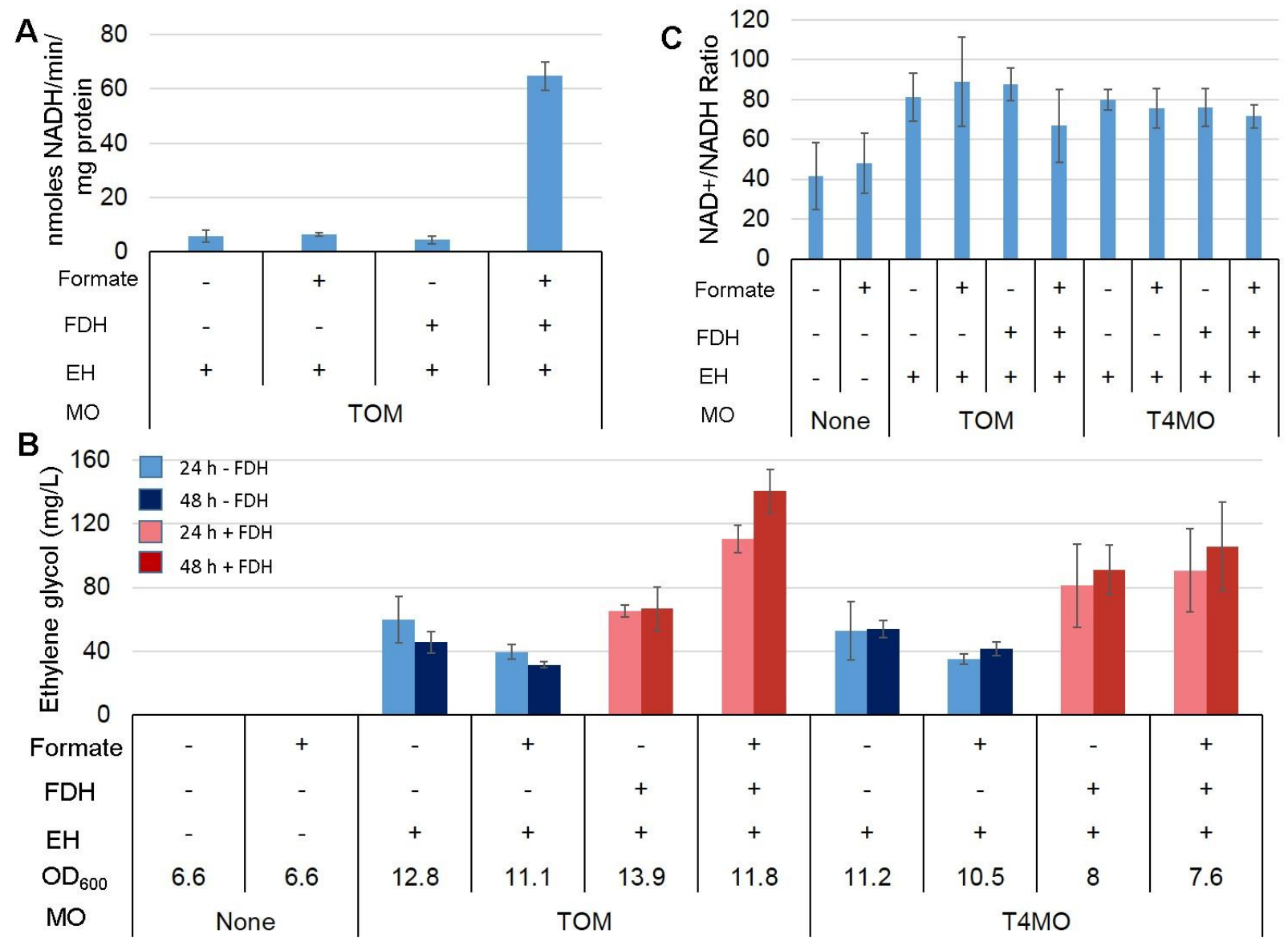

Figure 3. Ethylene glycol production with formate and FDH. A. FDH enzyme activity at 24

471 h. B. Strains, with and without FDH, and with and without supplemented formate were

472 monitored for ethylene glycol production over 48 h. C. Intracellular ratio of $\mathrm{NAD}^{+}$to NADH

473 concentrations after 48 h. EH, epoxide hydrolase; FDH, formate dehydrogenase; OD, optical

474 density at time zero; $\mathrm{MO}$, monooxygenase. $\mathrm{N}=3$; error bars represent standard deviations. 


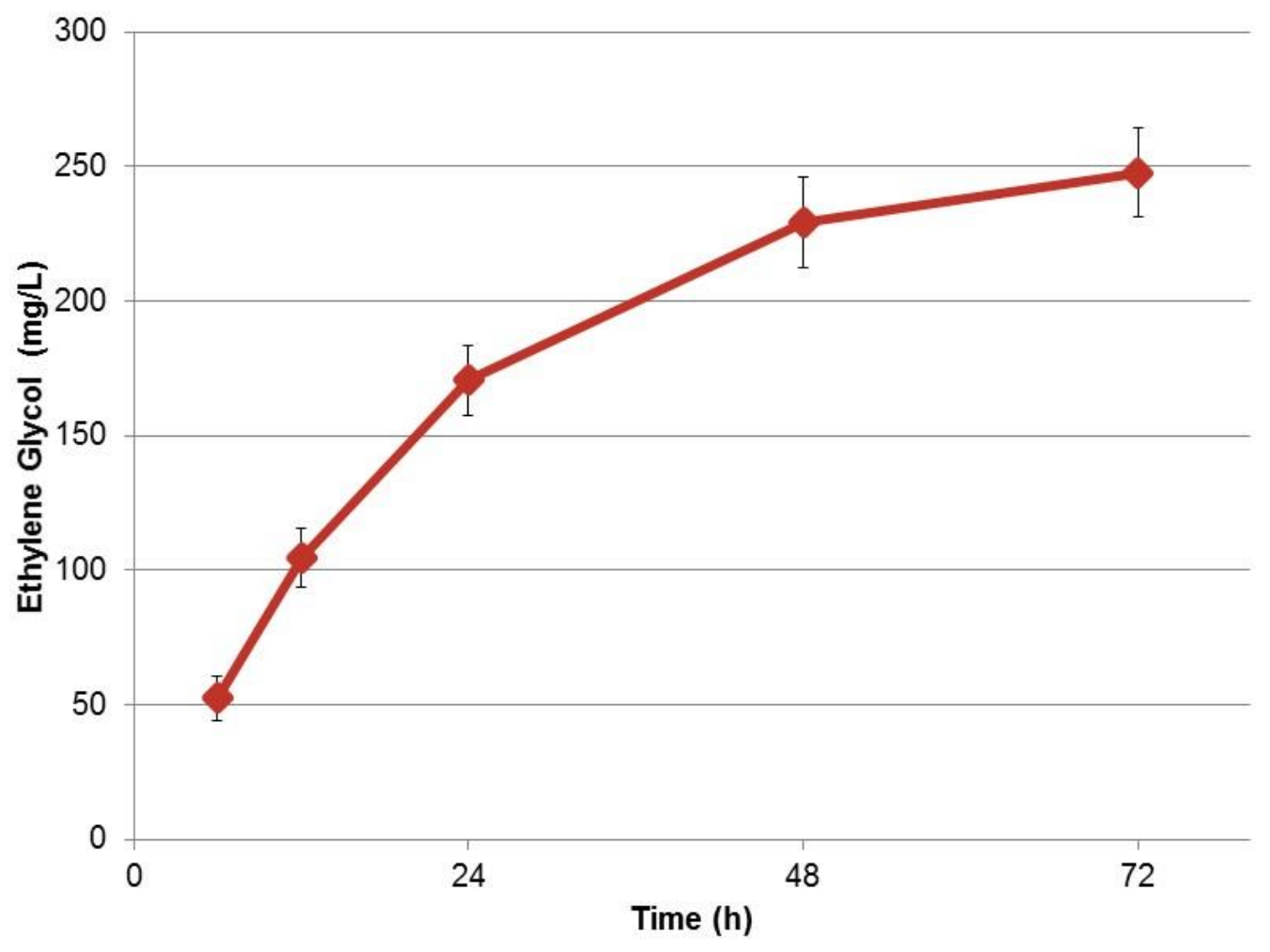

476

Figure 4. Ethylene glycol production from high cell densities. Ethylene glycol production

478 from Strain TOF by feeding $10 \mathrm{mM}$ formate and ethylene at $0,6,12,24,48$, and $72 \mathrm{~h}$. N=3; error 479 bars represent standard deviations. Starting $\mathrm{OD}_{600}$ was 57. 